\title{
XMM-Newton survey of the Local Group galaxy M $33^{\star, \star \star}$
}

\author{
W. Pietsch ${ }^{1}$, Z. Misanovic ${ }^{1}$, F. Haberl ${ }^{1}$, D. Hatzidimitriou ${ }^{2}$, M. Ehle ${ }^{3}$, and G. Trinchieri ${ }^{4}$ \\ 1 Max-Planck-Institut für extraterrestrische Physik, Giessenbachstraße, 85741 Garching, Germany \\ e-mail: wnp@mpe.mpg.de \\ 2 Department of Physics, University of Crete, PO Box 2208, 71003 Heraklion, Crete, Greece \\ 3 XMM-Newton Science Operations Centre, ESA, Villafranca del Castillo, PO Box 50727, 28080 Madrid, Spain \\ ${ }^{4}$ Osservatorio Astronomico di Brera, via Brera 28, 20121 Milano, Italy
}

Received 13 January 2004 / Accepted 4 March 2004

\begin{abstract}
In an XMM-Newton raster observation of the bright Local Group spiral galaxy M 33 we study the population of $\mathrm{X}$-ray sources (X-ray binaries, supernova remnants) down to a $0.2-4.5 \mathrm{keV}$ luminosity of $10^{35} \mathrm{erg} \mathrm{s}^{-1}$ - more than a factor of 10 deeper than earlier ROSAT observations. EPIC hardness ratios and optical and radio information are used to distinguish between different source classes. The survey detects 408 sources in an area of 0.80 square degree. We correlate these newly detected sources with earlier M 33 X-ray catalogues and information from optical, infra-red and radio wavelengths. As M 33 sources we detect 21 supernova remnants (SNR) and 23 SNR candidates, 5 super-soft sources and 2 X-ray binaries (XRBs). There are 267 sources classified as hard, which may either be XRBs or Crab-like SNRs in M 33 or background AGN. The 44 confirmed and candidate SNRs more than double the number of X-ray detected SNRs in M 33. 16 of these are proposed as SNR candidates from the X-ray data for the first time. On the other hand, there are several sources not connected to M 33: five foreground stars, 30 foreground star candidates, 12 active galactic nucleus candidates, one background galaxy and one background galaxy candidate. Extrapolating from deep field observations we would expect 175 to 210 background sources in this field. This indicates that about half of the sources detected are within M 33.
\end{abstract}

Key words. galaxies: individual: M 33 - X-rays: galaxies

\section{Introduction}

The Local Group Sc galaxy M 33 is located at a distance of $795 \mathrm{kpc}$ (van den Bergh 1991, i.e. 1" corresponds to $3.9 \mathrm{pc}$ and the flux to luminosity conversion factor is $7.6 \times 10^{49} \mathrm{~cm}^{2}$ ), is seen under a relatively low inclination of $56^{\circ}$ (Zaritsky et al. 1989) and the optical extent can be approximated by an inclination-corrected $D_{25}$ ellipse with large diameter of 64.4 and axes ratio of 1.66 (de Vaucouleurs et al. 1991; Tully 1988). With its moderate Galactic foreground absorption $\left(N_{\mathrm{H}}=\right.$ $6 \times 10^{20} \mathrm{~cm}^{-2}$, Stark et al. 1992) M 33 is ideally suited to study the X-ray source population and diffuse emission in a nearby spiral galaxy. The Einstein X-ray Observatory detected diffuse emission from hot gas in M 33 and 17 unresolved sources (Long et al. 1981; Markert \& Rallis 1983; Trinchieri et al. 1988). First ROSAT HRI and PSPC observations revealed 57 sources and confirmed the detection of diffuse X-ray emission which may trace the spiral arms within a $10^{\prime}$ radius around the nucleus (Schulman \& Bregman 1995; Long et al. 1996). Combining all archival ROSAT observations of the field,

* XMM-Newton is an ESA Science Mission with instruments and contributions directly funded by ESA Member States and the USA (NASA).

$\star \star$ Full Table 3 is only available in electronic form at the CDS via anonymous ftp to cdsarc.u-strasbg.fr $(130.79 .128 .5)$ or via http://cdsweb.u-strasbg.fr/cgi-bin/qcat?]/A+A/426/11
Haberl \& Pietsch (2001, hereafter HP01) found 184 X-ray sources within a $50^{\prime}$ radius around the nucleus, identified some of the sources by correlations with previous X-ray, optical and radio catalogues, and in addition classified sources according to their X-ray properties. They found candidates for super-soft X-ray sources (SSS), X-ray binaries (XRBs), supernova remnants (SNRs), foreground stars and active galactic nuclei (AGN).

To follow up on these findings, we proposed as part of the telescope scientist guaranteed time (GT) program a deep homogeneous XMM-Newton survey of M 33 with a sensitivity of $10^{35} \mathrm{erg} \mathrm{s}^{-1}$ in the $0.5-10 \mathrm{keV}$ band, a factor of ten deeper than previous surveys. The survey consisted of a raster of 15 pointings of about $10 \mathrm{ks}$ each. The directions were selected in a way that each area within the M 33 optical $D_{25}$ extent was covered by the XMM-Newton EPIC detectors at least three times with the medium filter. The survey was complemented by an additional AO2 program, initiated to fill observation gaps due to high detector background, or, because the thick filter was used during the GT observations. First results of this survey were presented in Pietsch et al. (2003a).

Two M 33 sources are known for their outstanding X-ray properties (Peres et al. 1989). The brightest source (X-8 in the nomenclature of Long et al. 1981, luminosity of $10^{39-40} \mathrm{erg} \mathrm{s}^{-1}$ ) is the most luminous X-ray source in 
Table 1. XMM-Newton M 33 observation log (proposal numbers 010264 and 014198).

\begin{tabular}{|c|c|c|c|c|c|c|c|c|c|c|}
\hline \multirow{3}{*}{$\begin{array}{c}\text { Field } \\
\text { M33 } \\
\text { (1) }\end{array}$} & \multirow{3}{*}{$\begin{array}{l}\text { Obs. id. } \\
\text { (2) }\end{array}$} & \multirow{3}{*}{$\begin{array}{c}\text { Obs. dates } \\
\text { (3) }\end{array}$} & \multirow{2}{*}{\multicolumn{2}{|c|}{$\begin{array}{l}\text { Pointing direction } \\
\text { RA/DEC (J2000) }\end{array}$}} & \multicolumn{2}{|c|}{ EPIC PN } & \multicolumn{2}{|c|}{ EPIC MOS1 } & \multicolumn{2}{|c|}{ EPIC MOS2 } \\
\hline & & & & & \multirow{2}{*}{ 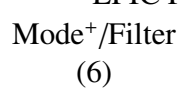 } & \multirow{2}{*}{$\begin{array}{c}T_{\exp }^{\dagger} \\
(7)\end{array}$} & \multirow{2}{*}{$\begin{array}{c}\text { Mode }{ }^{+} / \text {Filter } \\
(8)\end{array}$} & \multirow{2}{*}{$\begin{array}{c}T_{\text {exp }}^{\dagger} \\
(9)\end{array}$} & \multirow{2}{*}{$\begin{array}{c}\text { Mode }^{+} / \text {Filter } \\
\text { (10) }\end{array}$} & \multirow{2}{*}{$\begin{array}{l}T_{\text {exp }}^{\dagger} \\
(11)\end{array}$} \\
\hline & & & (4) & (5) & & & & & & \\
\hline 1 & 0102640101 & $2000-08-04$ & $1: 33: 51.0$ & $30: 39: 37$ & FF/medium & 6.29 & PW2/medium & 6.23 & PW2/medium & 6.22 \\
\hline 1 & 0141980501 & 2003-01-22 & $1: 33: 51.0$ & $30: 39: 37$ & $\mathrm{FF} /$ medium & 1.23 & FF/medium & 1.51 & FF/medium & 1.51 \\
\hline 1 & 0141980801 & $2003-02-12$ & $1: 33: 51.0$ & $30: 39: 37$ & $\mathrm{FF} /$ medium & 7.25 & $\mathrm{FF} /$ medium & 8.86 & $\mathrm{FF} /$ medium & 8.87 \\
\hline 2 & 0102640201 & 2000-08-04 & $1: 34: 40.0$ & $30: 57: 48$ & $\mathrm{EFF} /$ medium & 11.40 & $\mathrm{FF} /$ medium & 15.24 & $\mathrm{FF} /$ medium & 15.24 \\
\hline 3 & 0102640301 & 2000-08-07 & $1: 33: 32.0$ & $30: 52: 13$ & $\mathrm{EFF} /$ medium & 3.80 & $\mathrm{FF} /$ medium & 4.58 & $\mathrm{FF} /$ thin & 4.58 \\
\hline 3 & 0141980401 & 2003-01-24 & $1: 33: 32.0$ & $30: 52: 13$ & $\mathrm{FF} /$ medium & 0.00 & $\mathrm{FF} /$ medium & 0.00 & $\mathrm{FF} /$ medium & 0.00 \\
\hline 4 & 0102640401 & 2000-08-02 & $1: 32: 51.0$ & $30: 36: 49$ & $\mathrm{EFF} /$ thick & 8.66 & $\mathrm{FF} /$ thick & 12.99 & $\mathrm{FF} /$ thick & 12.99 \\
\hline 4 & 0141980601 & $2003-01-23$ & $1: 32: 51.0$ & $30: 36: 49$ & $\mathrm{FF} /$ medium & 11.18 & $\mathrm{FF} /$ medium & 12.56 & $\mathrm{FF} /$ medium & 12.56 \\
\hline 5 & 0102640501 & 2001-07-05 & $1: 33: 02.0$ & $30: 21: 24$ & $\mathrm{FF} /$ medium & 9.26 & $\mathrm{FF} /$ medium & 11.76 & $\mathrm{FF} /$ medium & 11.76 \\
\hline 6 & 0102640601 & 2001-07-05 & $1: 34: 08.0$ & $30: 46: 06$ & $\mathrm{FF} /$ medium & 2.86 & $\mathrm{FF} /$ medium & 3.42 & $\mathrm{FF} /$ medium & 3.42 \\
\hline 6 & 0141980301 & $2003-07-25$ & $1: 34: 08.0$ & $30: 46: 06$ & $\mathrm{FF} /$ medium & 4.12 & $\mathrm{FF} /$ medium & 4.82 & $\mathrm{FF} /$ medium & 4.82 \\
\hline 7 & 0102640701 & 2001-07-05 & 1:34:10.0 & $30: 27: 00$ & $\mathrm{FF} /$ medium & 6.97 & $\mathrm{FF} /$ medium & 10.96 & $\mathrm{FF} /$ medium & 10.95 \\
\hline 8 & 0102640801 & 2001-07-07 & $1: 34: 51.0$ & $30: 42: 22$ & $\mathrm{FF} /$ medium & - & $\mathrm{FF} /$ medium & 1.19 & $\mathrm{FF} /$ medium & 1.19 \\
\hline 8 & 0102642001 & 2001-08-15 & $1: 34: 51.0$ & $30: 42: 22$ & $\mathrm{FF} /$ medium & 8.65 & $\mathrm{FF} /$ medium & 11.15 & $\mathrm{FF} /$ medium & 11.15 \\
\hline 9 & 0102640901 & 2001-07-07 & $1: 34: 04.0$ & $30: 57: 25$ & $\mathrm{FF} /$ medium & 2.42 & $\mathrm{FF} /$ medium & 3.15 & $\mathrm{FF} /$ medium & 3.15 \\
\hline 9 & 0141980201 & 2003-07-11 & 1:34:04.0 & $30: 57: 25$ & $\mathrm{FF} /$ medium & 3.74 & $\mathrm{FF} /$ medium & 4.02 & $\mathrm{FF} /$ medium & 4.02 \\
\hline 10 & 0102641001 & 2001-07-08 & 1:33:07.0 & $30: 45: 02$ & $\mathrm{FF} /$ medium & 1.25 & $\mathrm{FF} /$ medium & 8.33 & $\mathrm{FF} /$ medium & 8.37 \\
\hline 10 & 0141980101 & $2003-07-11$ & $1: 33: 07.0$ & $30: 45: 02$ & $\mathrm{FF} /$ medium & 5.45 & $\mathrm{FF} /$ medium & 5.75 & $\mathrm{FF} /$ medium & 5.77 \\
\hline 11 & 0102641101 & 2001-07-08 & $1: 32: 46.0$ & $30: 28: 19$ & $\mathrm{FF} /$ medium & 8.10 & $\mathrm{FF} /$ medium & 10.36 & $\mathrm{FF} /$ medium & 10.36 \\
\hline 12 & 0102641201 & 2000-08-02 & $1: 33: 38.0$ & $30: 21: 49$ & EFF/thick & 11.34 & FF/thick & 3.67 & $\mathrm{FF} /$ thick & 3.66 \\
\hline 12 & 0141980701 & 2003-01-24 & $1: 33: 38.0$ & $30: 21: 49$ & $\mathrm{FF} /$ medium & 4.40 & $\mathrm{FF} /$ medium & 5.72 & $\mathrm{FF} /$ medium & 5.73 \\
\hline 13 & 0102642101 & $2002-01-25$ & $1: 34: 34.0$ & $30: 34: 11$ & $\mathrm{FF} /$ medium & 10.00 & $\mathrm{FF} /$ medium & 12.27 & $\mathrm{FF} /$ medium & 12.27 \\
\hline 14 & 0102642201 & $2002-01-25$ & $1: 34: 56.0$ & $30: 50: 52$ & $\mathrm{FF} /$ medium & 11.60 & $\mathrm{FF} /$ medium & 13.87 & $\mathrm{FF} /$ medium & 13.87 \\
\hline 15 & 0102642301 & 2002-01-27 & $1: 33: 33.0$ & $30: 33: 07$ & $\mathrm{FF} /$ medium & 10.00 & $\mathrm{FF} /$ medium & 12.27 & $\mathrm{FF} /$ medium & 12.27 \\
\hline
\end{tabular}

Notes:

${ }^{+}$: FF: full frame, EFF: extended full frame, PW2: partial window 2.

$\dagger$ : Exposure time in units of ks after screening for high background (see text).

the Local Group of galaxies and coincides with the optical center of M 33. Its time variability (Dubus et al. 1997), its point-like nature seen by ROSAT HRI (Pietsch \& Haberl 2000) and Chandra (Dubus \& Rutledge 2002) and its X-ray spectrum best described by an absorbed power law plus disk blackbody model (e.g., Ehle et al. 2001; La Parola et al. 2003; Foschini et al. 2004), point towards a black hole XRB. A possible periodicity of 106 days was not confirmed in later observations (see Parmar et al. 2001). The second source (X-7) is an eclipsing high mass XRB (HMXB) with a binary period of $3.45 \mathrm{~d}$ and possible $0.31 \mathrm{~s}$ pulsations, discovered in ROSAT PSPC and HRI observations (Schulman et al. 1993; Dubus et al. 1997, 1999; Larson \& Schulman 1997). Pietsch et al. (2004) analyzed XMM-Newton and Chandra data and determined improved binary parameters, however, they could not confirm the proposed pulsations. In a special analysis of DIRECT data, they identified an O7I star of 18.89 mag in V, as the optical counterpart, which shows the ellipsoidal heating light curve of a HMXB with the X-7 binary ephemeris. X-7 was the most distant eclipsing XRB until the detection of another such source in XMM-Newton and Chandra observations of the starburst galaxy NGC 253, which is located at more than three times the distance of M 33 (Pietsch et al. 2003b).

Here we present merged medium and thin filter images for the three EPIC instruments, in five energy bands, using only times of low background from our XMM-Newton survey of M 33, as well as a source catalogue and source population study based on these images. An analysis of the individual pointings with less stringent background rejection and using all filters is in progress, and will result in a source catalogue with improved positions (taking care of individual pointing offsets), that will also provide information on source variability and spectral characteristics.

\section{Observations and data analysis}

Table 1 summarizes the XMM-Newton (Jansen et al. 2001) EPIC (Strüder et al. 2001; Turner et al. 2001) observations. For each observation we give the field number of our M 33 raster (Col. 1), the observation identification (2), date (3), pointing direction $(4,5)$, as well as instrument mode, filter and exposure time after screening for high background for EPIC PN $(6,7), \operatorname{MOS} 1(8,9)$, and MOS2 $(10,11)$. Observation 0102640801 was affected by a satellite "slew failure" and only the EPIC MOS instruments got some exposure time.

In the XMM-Newton observations the EPIC PN and MOS instruments were mostly operated in the full frame mode resulting in a time resolution of $73.4 \mathrm{~ms}$ and $2.6 \mathrm{~s}$, respectively. Only for four early observations was the PN detector operated in the extended full frame mode (time resolution $200 \mathrm{~ms}$ ). During observation 0102640101 the MOS detectors were operated in the small window mode $(0.3 \mathrm{~s}$ time resolution for the inner CCDs to avoid pile-up for the bright source $\mathrm{X}-8$ ). The medium filter was in front of the EPIC cameras in all but 
the first two observations which were performed with the thick filter (0102640401, 0102641201) and observation 0102640301 for which the MOS2 detector was operated with the thin filter. For creating the merged images and for source detection, we only used medium and thin filter observations.

The data analysis was performed using tools in the XMMNewton Science Analysis System (SAS) v5.4.1 and some later versions from the development area as specially mentioned, EXSAS/MIDAS 1.2/1.4, and FTOOLS v5.2 software packages, the imaging application DS9 v2.3b1 and the spectral analysis software XSPEC v11.2.

For MOS, we used the SAS task emchain with task emevent v7.10 that removes flickering pixels. We carefully screened the event files for bad CCD pixels, remaining after the standard processing. To create a homogeneous combined image with similar background level for all fields we had to carefully screen the data for high background periods. In most observations the background light curves at all energies follow the high energy (7-15 keV) background light curves provided by the SAS tasks epchain and emchain and allowed us to efficiently screen the data. In some observations, however, the low energy background (below $1 \mathrm{keV}$ ) was higher during high energy selected good time intervals (GTIs) and had to be used for additional screening. GTIs were determined from the higher signal-to-noise PN light curves and also used for the MOS cameras. Outside the PN time coverage, GTIs were determined from the combined MOS light curves. The corresponding low background times for the individual observations are listed for the PN and MOS cameras in Table 1. The combined "PN equivalent" vignetted EPIC exposure (1-2 keV) is significantly above $20 \mathrm{ks}$ within most of the optical extent of M 33 (indicated by the inclination-corrected $D_{25}$ ellipse in Fig. 1). Only in the NW and S the exposure does not reach this limit. For the "PN equivalent" EPIC exposure map we added MOS1 and MOS2 exposure maps (scaled by a factor of 0.36 as expected for a $5 \mathrm{keV}$ thermal bremsstrahlung spectrum with an absorption of $6 \times 10^{20} \mathrm{~cm}^{-2}$, as expected for XRBs in M 33) to the PN exposure.

For the analysis we used five energy bands: $(0.2-0.5) \mathrm{keV}$, (0.5-1.0) keV, (1.0-2.0) keV, (2.0-4.5) keV, and (4.5-12) keV as band 1 to 5 . We intentionally split the $0.5-2.0 \mathrm{keV}$ band used in the 1XMM XMM-Newton Serendipitous Source Catalogue ${ }^{1}$ to get, on average, a more homogeneous distribution of the source counts to the energy bands, which leads to a better spread of the hardness ratio values and allows effective source classification (see Sect. 6).

For PN we selected only "singles" (PATTERN = 0) in band 1, for the other bands "singles and doubles" (PATTERN $\leq 4)$. For MOS we used "singles" to "quadruples" (PATTERN $\leq 12$ ). To avoid background variability over the PN images we omitted the energy range (7.2-9.2) $\mathrm{keV}$ in band 5 where strong fluorescence lines cause higher background in the outer detector area (Freyberg et al. 2004). To convert source count rates in the individual bands to fluxes we calculated count rate to energy conversion factors (ECF) for PN and MOS

\footnotetext{
1 Prepared by the XMM-Newton Survey Science Centre Consortium (http://xmmssc-www.star.le.ac.uk/).
}

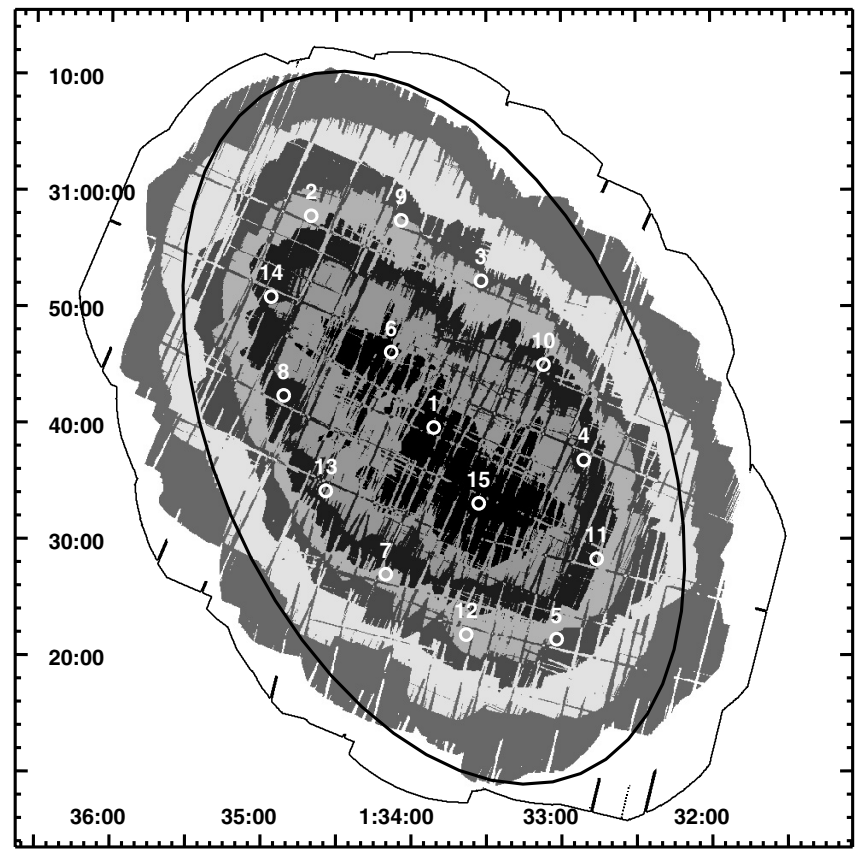

Fig. 1. XMM-Newton EPIC "PN equivalent" exposure map combining all low background observation times of the fields of the M 33 raster. The outer contour indicates $0 \mathrm{ks}$, gray-scale steps every $10 \mathrm{ks}$. The maximum PN equivalent exposure (see text) is $89 \mathrm{ks}$ in the center area of M 33. The optical extent of M 33 is marked by the white inclination-corrected $D_{25}$ ellipse. The center of the fields of our raster (see Table 1) are indicated and numbered.

Table 2. Count rate to energy conversion factor for different spectral models for EPIC instruments in energy band 1 to 5 (B1-B5). Models are a power law with photon index of 1.7 (PL), a thin thermal model with temperature of $1 \mathrm{keV}$ (TT), and a black body with a temperature of $30 \mathrm{eV}(\mathrm{BB})$. All models assume galactic foreground absorption of $6 \times 10^{20} \mathrm{~cm}^{-2}$ (Stark et al. 1992).

\begin{tabular}{lcrrrrr}
\hline \hline Detector & Spec. & $B 1$ & $\begin{array}{c}B 2 \\
\left(10^{-12} \mathrm{erg} \mathrm{cm}^{-2} \mathrm{ct}^{-1}\right)\end{array}$ & $B 5$ \\
\hline EPIC PN & PL & 1.03 & 1.10 & 1.71 & 4.9 & 21. \\
& TT & 1.03 & 1.14 & 1.55 & 4.0 & \\
& BB & 1.09 & 0.97 & 1.30 & & \\
EPIC MOS & PL & 6.2 & 4.9 & 4.8 & 12.9 & 66. \\
& TT & 6.0 & 4.5 & 4.6 & 11.0 & \\
& BB & 10.1 & 4.8 & 4.2 & & \\
\hline
\end{tabular}

medium filter observations using W3PIMMS and assuming the same spectrum as for the first XMM-Newton source catalogue, i.e. a power law spectrum with photon index 1.7 absorbed by the galactic foreground column of $6 \times 10^{20} \mathrm{~cm}^{-2}$ (Table 2). The assumed spectrum represents hard sources like XRBs or AGN. We also give ECF values for a typical SNR (absorbed $1 \mathrm{keV}$ thin thermal) and SSS (absorbed $30 \mathrm{eV}$ black body) in M 33. Table 2 demonstrates that for EPIC PN, ECF values only vary by about $20 \%$ for the different spectra in bands 1 to 4 . The same is true for EPIC MOS, with the exception of the black body ECF in the $0.2-0.5 \mathrm{keV}$ band where the MOS sensitivity is much lower. For both PN and MOS, the sensitivity is 
strongly reduced at energies above $4.5 \mathrm{keV}$. On the other hand, background count rates are similar in the hard band. Therefore, only bright hard sources are detected in this band.

To classify the source spectra we computed four hardness ratios from the source count rates. These hardness ratios are defined as $H R i=\left(B_{i+1}-B_{i}\right)\left(B_{i+1}+B_{i}\right)$, for $i=1$ to 4 , where $B_{i}$ denotes count rates in band $i$, as defined above. In the standard source detection products, hardness ratios and count rates in individual energy bands are not combined for all instruments. To improve the statistics, we have combined the count rates (and fluxes) from all three EPIC instruments and have then derived hardness ratios for them. We are aware that these products have some jitter in their meaning, as the relative integration times for individual sources in the EPIC instruments differ slightly. Due to the better signal in the combined products, they are still very valuable for source classification. For investigations needing accurate calibration, values derived for each instrument separately should be used.

As discussed above, band 5 suffers from the combined effect of lower sensitivity in both PN and MOS, and a large contamination from the hard background, reducing the significance of any detection due to the much higher noise. We therefore give total count rates and errors derived from all instruments in the "XID" band that comprises bands 1-4, and derive fluxes in the corresponding $0.2-4.5 \mathrm{keV}$ range. A larger band would artificially increase the overall noise of all but extremely hard sources. For very soft sources, or for sources detected only in one of the instruments (e.g. soft sources in PN), even with this choice the quoted errors are overestimated, however the number of sources of this nature is small.

For PN, MOS1 and MOS2 we created images, background images, exposure maps (without and with vignetting correction) and masked them for the acceptable detector area in each of the five energy bands. For PN, the background maps contain the contribution from the "out of time (OOT)" events (parameter withootset=true in task esplinemap). The images of the individual observations were then merged for source detection into images covering the full M 33 field. The maximum vignetted exposure was $44.3 \mathrm{ks}, 58.6 \mathrm{ks}$ and $58.7 \mathrm{ks}$ in the 1-2 keV band for the PN, MOS1 and MOS2 cameras, respectively, and was nearly the same for bands 1 to 4 . In the hardest band, the maximum exposure is smaller by $30 \%$ due to the larger vignetting effects. To allow us an easy merging of images, we calculated for the events of all observations projected sky coordinates $(X, Y)$ with respect to the center reference position RA $=01^{\mathrm{h}} 33^{\mathrm{m}} 50^{\mathrm{s}} \cdot 4$, Dec $=+30^{\circ} 39^{\prime} 36^{\prime \prime}(\mathrm{J} 2000)$. At the borders, due to the different field of view of the EPIC instruments, the merged images for PN, MOS1 and MOS2 do not fully overlap. Nevertheless, we used the full area for source detection.

\section{Source catalogue}

We searched for sources in these merged images simultaneously using $5 \times 3$ images ( 5 energy bands and PN, MOS1 and MOS2 camera). A preliminary source list created with the task boxdetect with a low likelihood threshold was used as starting point for the task emldetect v4.31. As photons collected with different off-axis angles contribute to the source counts in the merged image, we prepared a specific point spread function (PSF) calibration file. It assumed a constant PSF over the total field of view that was degraded as if $6^{\prime}$ off-axis. This is the most degraded but still "circular" PSF in the EPIC calibration files and a first approximation for the PSF when source counts are collected at many different off-axis angles as in the M 33 raster observation. To resolve sources that overlap due to the PSF, we used parameter multisourcefit $=4$.

A detection probability above $4 \sigma$ is equivalent to a single band limiting detection likelihood of 10. This threshold has been successfully used by HP01 for the ROSAT PSPC detections (see Sect. 5). In the case of simultaneous detection with a large number of images this equivalence is no longer valid due to the small number of counts in the individual images contributing to a source at the detection threshold. We have established for the task emldetect that for the case of simultaneously using 15 images, a detection probability above $4 \sigma$ corresponds to a combined likelihood of 7 . This threshold was used to select sources for the catalogue. The detection procedure produced acceptable results as can be checked from the overlay of the detected sources onto the smoothed X-ray images (see Sect. 4).

Two emission peaks were resolved into several sources with no sign of multiplicity from the smoothed images and are better described as extended. In a detection run including extent determination we found that these two sources (\#125 and \#299) can be characterized by a Gaussian with FWHM of $18^{\prime \prime} .0$ and 16 .'2, respectively, and the extent likelihood is high. In total we detected 408 sources in the field.

The source parameters are summarized in Table 3 (EPIC combined products and products for EPIC PN, MOS1 and MOS2, separately). We only show the first five and the last row in the paper. The full table is available in electronic form at the CDS.

Table 3 gives the source number (Col. 1), position (2 to 8) with $1 \sigma$ uncertainty radius (9), likelihood of existence (10), integrated PN, MOS1 and MOS2 count rate and error $(11,12)$ and flux and error $(13,14)$ in the $0.2-4.5 \mathrm{keV}$ band, and hardness ratios and errors (15-22). Hardness ratios are calculated only for sources for which at least one of the two band count rates has a significance greater than $2 \sigma$. Errors are the properly combined statistical errors in each band and can extend beyond the range of allowed values of hardness ratios as defined previously ( -1.0 to 1.0$)$. The EPIC instruments contributing to the source detection, are indicated in the "Val" parameter (Col. 23, first character for PN, second MOS1, third MOS2) as "T", if inside the field of view (FOV), or "F", if outside of FOV. There are only nine sources at the periphery of the FOV where only part of the EPIC instruments contribute. The positional error does not include intrinsic systematic errors which, according to the Users Guide of the 1XMM XMM-Newton Serendipitous Source Catalogue, amount to 1.'5 and should be quadratically added to the statistical errors in Col. 9.

Table 3 then gives for EPIC PN exposure (24), source existence likelihood $(25)$, count rate and error $(26,27)$ and flux and error $(28,29)$ in the $0.2-4.5 \mathrm{keV}$ band, and hardness ratios and error (30-37). Columns 38 to 51 and 52 to 65 give 
Table 3. X-ray source catalogue of the XMM-Newton EPIC M 33 raster observation.

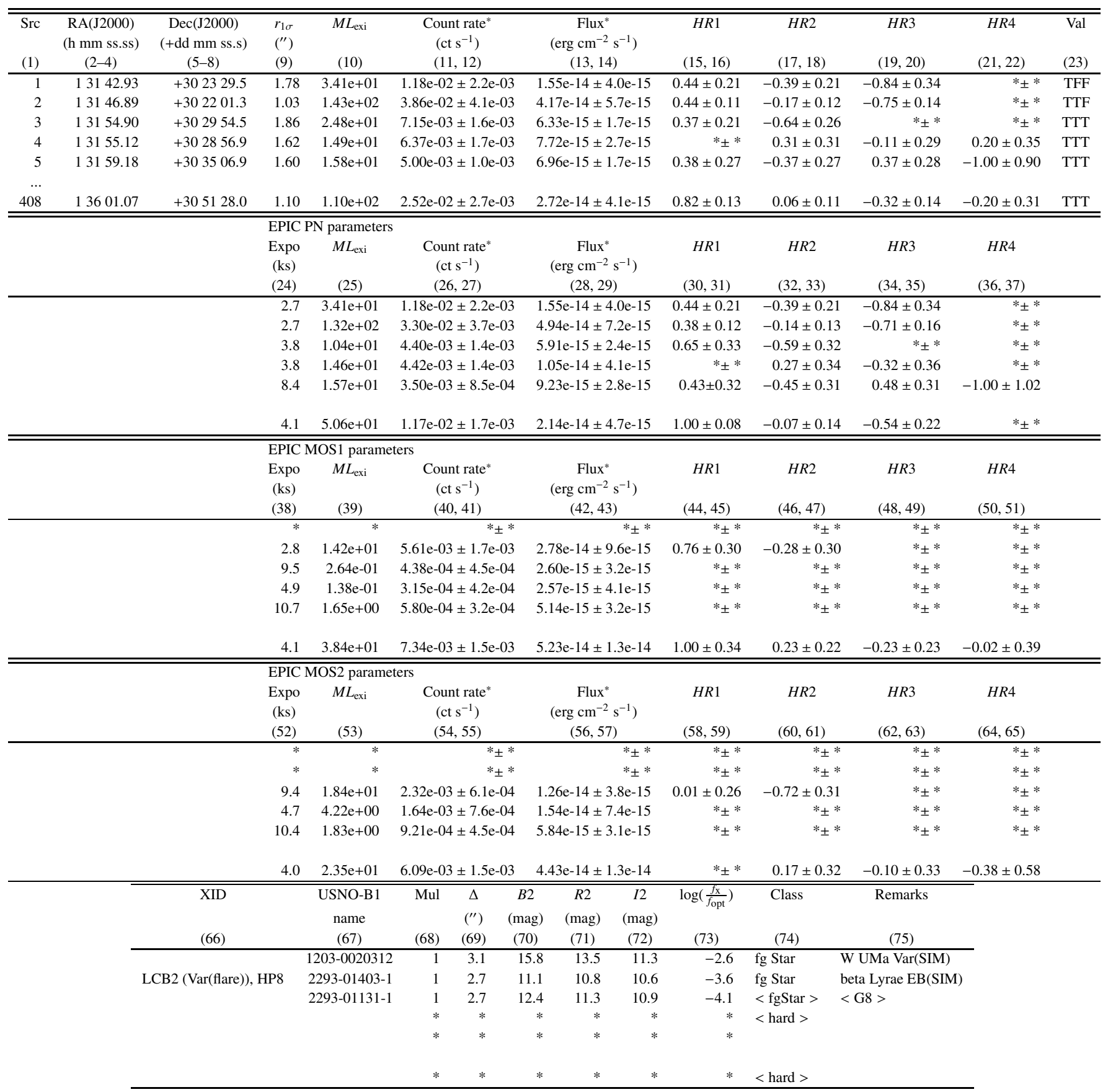

Notes:

*: In XID band $(0.2-4.5 \mathrm{keV})$.

References:

in XID: X-n: Long et al. (1981), Trinchieri et al. (1988); SBn: Schulman \& Bregman (1995); LCBn: Long et al. (1996); DCL97: Dubus et al. (1997); DCL99: Dubus et al. (1999); HPn: Haberl \& Pietsch (2001)

in remarks: SIM: SIMBAD database, NED: NASA Extragalactic Database, NVSS: Condon et al. (1998), CS82: Christian \& Schommer (1982), [MC83]25: Massey \& Conti (1983), IFM*: Ivanov et al. (1993), GKLn: Gordon et al. (1998), GDKn: Gordon et al. (1999), IV74: Israel \& van der Kruit (1974), VBW78: von Kap-Herr et al. (1978), M98: Massey (1998), MCM2001: Magrini et al. (2001), MBH96: Massey et al. (1996), PMM2004: Pietsch et al. (2004), GM94: Giovagnoli \& Mould (1994), FSZ: Fabrika et al. (1997), D33: Macri et al. (2001), Mochejska et al. (2001), RW93: Regan \& Wilson (1993), VHK: Hubble (1926), CGC97: Crampton et al. (1997), BCLMP: Boulesteix et al. (1974).

the same information corresponding to Cols. 24 to 37 , but now for the EPIC MOS1 and MOS2 instruments. Hardness ratios for the individual instruments were again screened as described above. From the comparison of the hardness ratios derived from integrated $\mathrm{PN}, \mathrm{MOS} 1$ and MOS2 count rates (Cols. 15-22) and the hardness ratios of the individual instruments (Cols. 30-37, 44-51 and 58-65) it is clear that combining the instrument count rate information yielded 


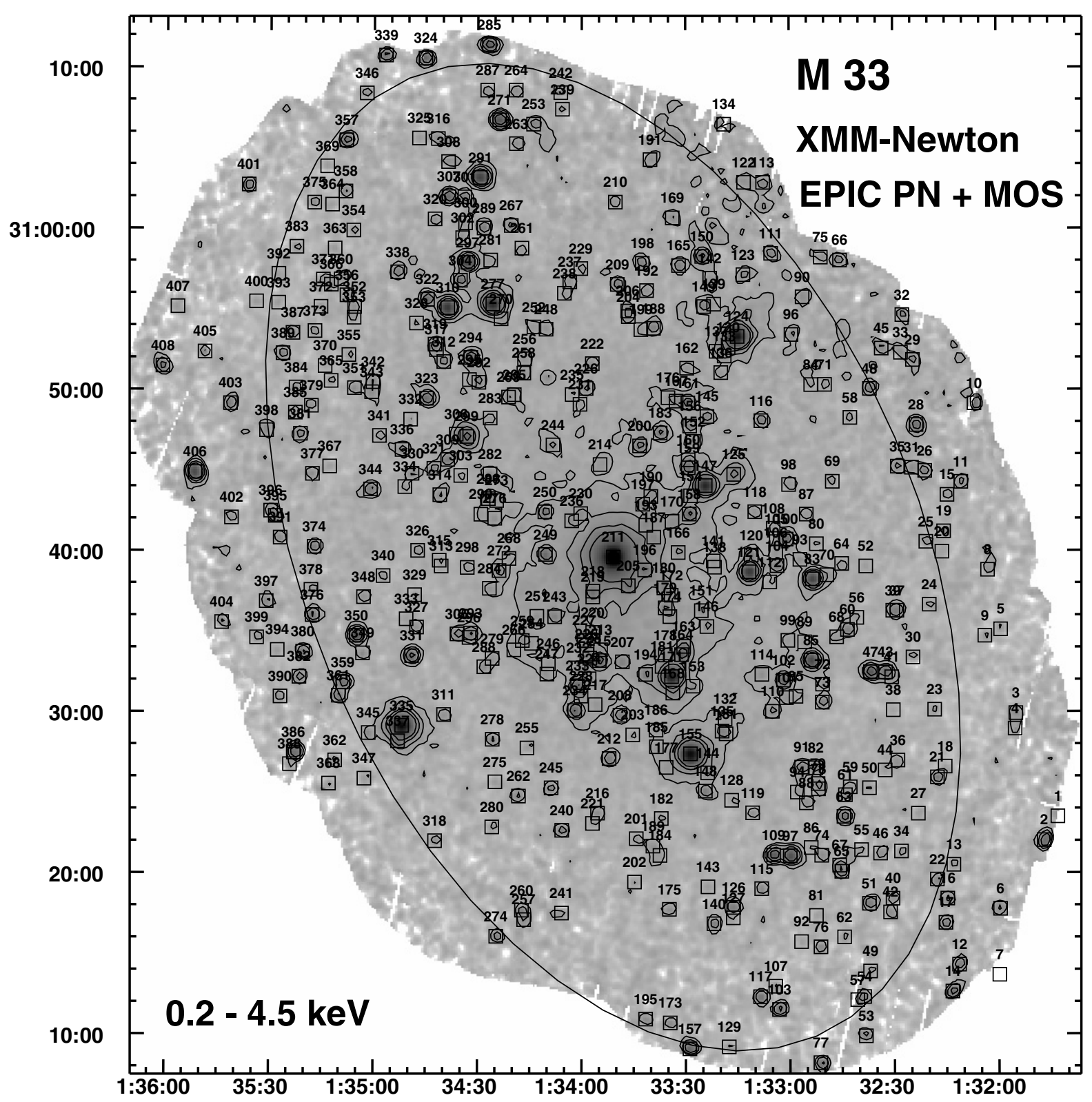

Fig. 2. Logarithmically-scaled XMM-Newton EPIC low background image of the M 33 medium and thin filter observations combining PN and MOS1 and MOS2 cameras in the (0.2-4.5) keV band. The data are smoothed with a Gaussian of FWHM 20" which corresponds to the average point spread function of the combined observations with different off-axis angles. The image is corrected for un-vignetted exposure and masked for exposure above $14 \mathrm{ks}$. Contours are at $(0.8,1.2,2.0,3.6) \times 10^{-5} \mathrm{ct} \mathrm{s}^{-1}$. Sources from the catalogue, RA, Dec (J2000.0) coordinates, and the optical $D_{25}$ ellipse of M 33 are marked.

significantly more hardness ratios above the chosen significance threshold.

In the remaining columns of Table 3 , we give cross correlation information, which is further described in Sect. 5. We only want to mention here, that we used the foreground stars and candidates, to verify the assumed source position errors. All 35 foreground stars and candidates are located within the $3 \sigma$ statistical plus systematic positional error given above.

The faintest sources detected have a flux of $1.0 \times$ $10^{-15} \mathrm{erg} \mathrm{cm}^{-2} \mathrm{~s}^{-1}$, the brightest source $(\mathrm{X}-8) 1.1 \times$ $10^{-11} \mathrm{erg} \mathrm{cm}^{-2} \mathrm{~s}^{-1}$ in the $0.2-4.5 \mathrm{keV}$ band. This corresponds to an absorbed luminosity range in M 33 of $7.6 \times 10^{34} \mathrm{erg} \mathrm{s}^{-1}$ to $8.4 \times 10^{38} \mathrm{erg} \mathrm{s}^{-1}$.

\section{Images}

For Figs. 2, 3 and 4 we smoothed the images and un-vignetted exposure maps per band and observation (created as described in Sect. 2) with a Gaussian ( $F W H M$ of $\left.20^{\prime \prime}\right)$ and masked them for acceptable detector area. For PN, we subtracted OOT images, that were masked and smoothed in the same way as the images. We normalized the images with the un-vignetted exposure maps to avoid an over-correction of the partly unvignetted detector background. For the EPIC combined images, we added the images of the individual cameras scaled according to the background in the individual energy bands. For the colour image and the broad band image we added images for 

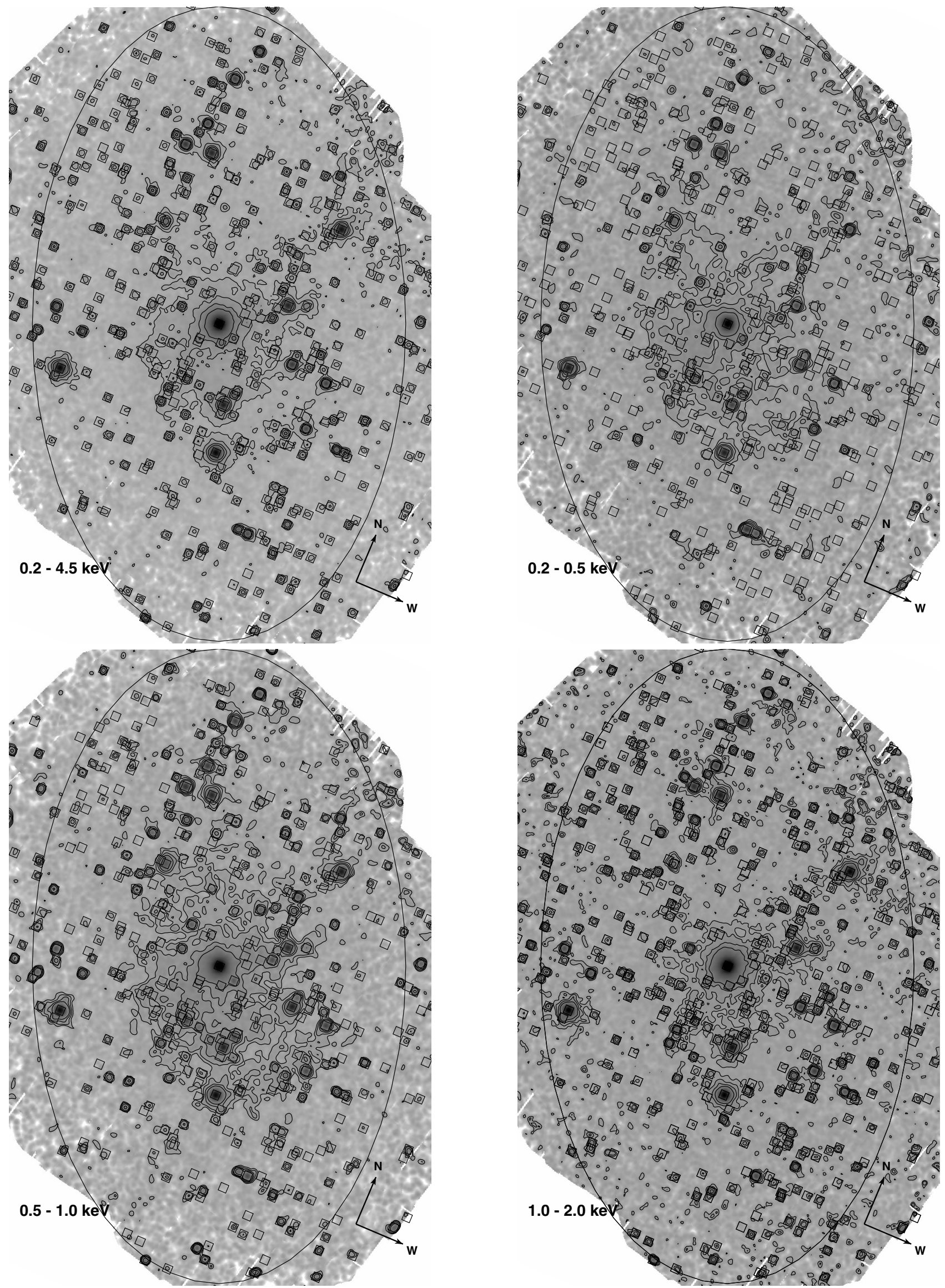

Fig. 3. XMM-Newton EPIC M 33 images: band 1-4 (upper left), band 1 (upper right), band 2 (lower left), and band 3 (lower right). 

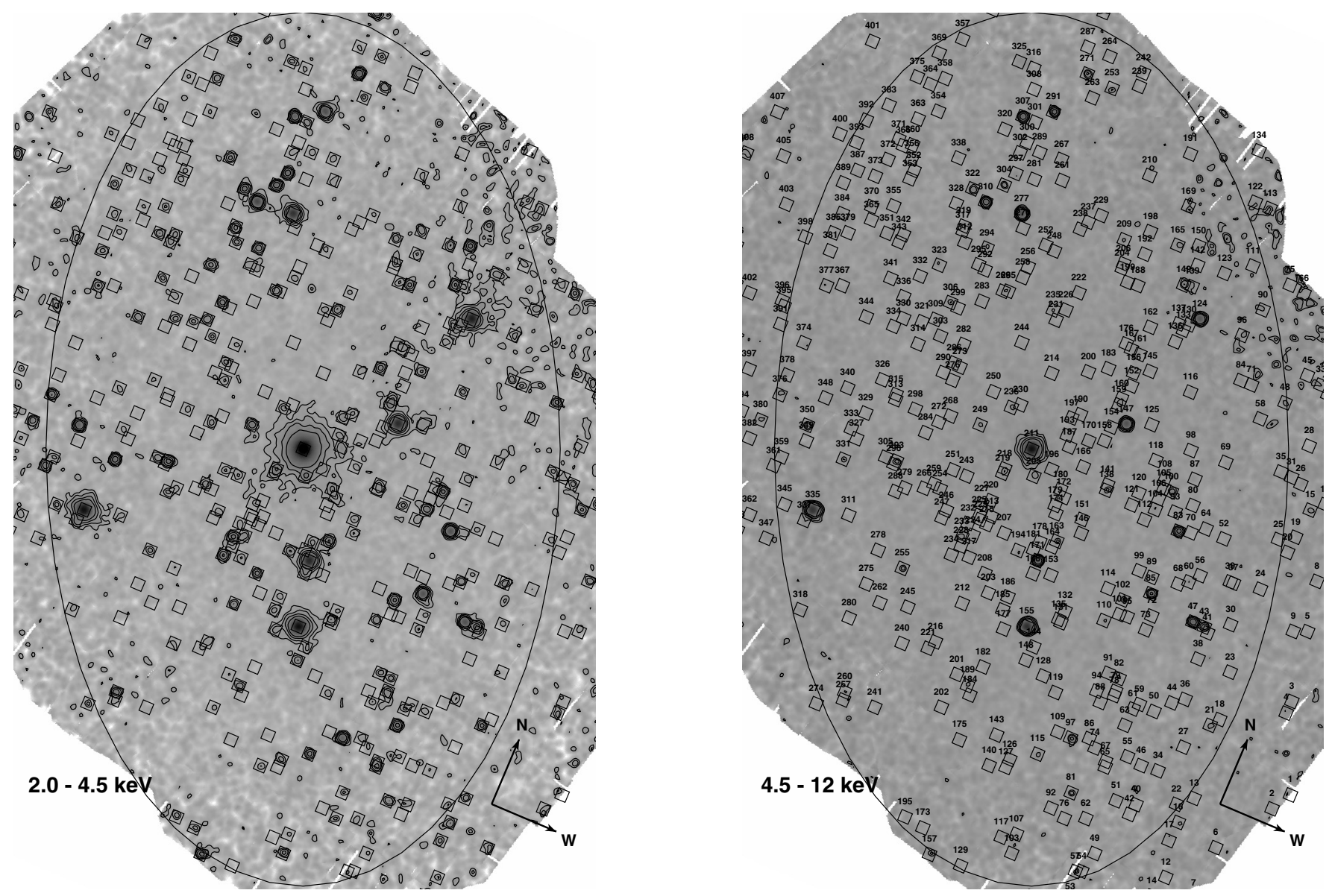

Fig. 3. (continued). XMM-Newton EPIC M 33 images: band 4 (left) and band 5 (right). The images are smoothed with a Gaussian of FWHM 20", corrected for un-vignetted exposure and masked for exposure above 14 ks. Contour levels are $(8,12,20,36) \times 10^{-6} \mathrm{ct} \mathrm{s}^{-1}$ for the merged band $1-4,(1.5,2,3,5) \times 10^{-6} \mathrm{ct} \mathrm{s}^{-1}$ for the band $1,(1.7,2.2,3.2,5.2) \times 10^{-6} \mathrm{ct} \mathrm{s}^{-1}$ for the band $2,(3,4,6,10) \times 10^{-6} \mathrm{ct} \mathrm{s}^{-1}$ for the band $3,(2.5,3.5,5.5,9.5) \times 10^{-6} \mathrm{ct} \mathrm{s}^{-1}$ for the band 4 , and $(5,6,8,12) \times 10^{-6} \mathrm{ct} \mathrm{s}^{-1}$ for the band 5 images. The inclination-corrected optical $D_{25}$ ellipse of M 33, the sky orientation, and the sources from the catalogue - including source numbers in the (4.5-12) keV band image - are indicated.

the individual bands as needed and masked them to a total unvignetted exposure of $10 \mathrm{ks}$. To better visualize faint structures we add contours. We indicate the detected sources by boxes and the optical extent of M 33 by the inclination-corrected $D_{25}$ ellipse.

The XID band (0.2-4.5 keV) image (Fig. 2) gives an overview on the sources detected in the XMM-Newton analysis. Two of the sources ( 1 and 7) are bright sources detected in areas with low exposure that were masked out for the images. In Fig. 3 we rotated the images of the individual band so that the major axis of the $D_{25}$ ellipse is oriented vertically. We overplotted the positions of the sources of our catalogue. By comparing the different energy bands it is clear that many sources only show up in some of the images, a fact that indicates spectral diversity and is further quantified in the different hardness ratios of the sources. This fact can be visualized even more clearly in the combined EPIC colour image where we coded the $0.2-1.0 \mathrm{keV}$ band in red, $1.0-2.0 \mathrm{keV}$ band in green and above $2.0 \mathrm{keV}$ in blue (see Fig. 4). The image is a demonstration of the colourful X-ray sky. SSS, thermal SNRs and foreground stars appear red or yellow, XRBs, Crab-like SNRs and AGN green to blue. Diffuse emission fills the inner area of the disk and the southern spiral arm (red, see also Fig. 3, band 1-3). A detailed analysis of this emission is outside the scope of this paper.

The images reveal that there is additional emission that has not been resolved into individual sources. This may be due to really diffuse emission or due to sources that did not reach the detection threshold. Examples are, structures in the area around the three bright sources X-9a,b,c $(277,297,310)$ in the NE, source X-4 (124) in the NW and source X-6 (155) in the SSW. There seems to be a relatively bright source which was missed by the detection procedure between sources 235 and 258. Faint structure appears between sources 55 and 65 and south of source 91 . Only deeper and/or higher resolution observations will allow us to clarify the nature of this emission.

\section{Cross-correlation with other M 33 X-ray catalogues}

In this section we discuss the cross-correlation of the XMMNewton detected sources with sources reported in earlier X-ray catalogues. All correlations (together with other X-ray information like variability, reported in these catalogues, or extent, 


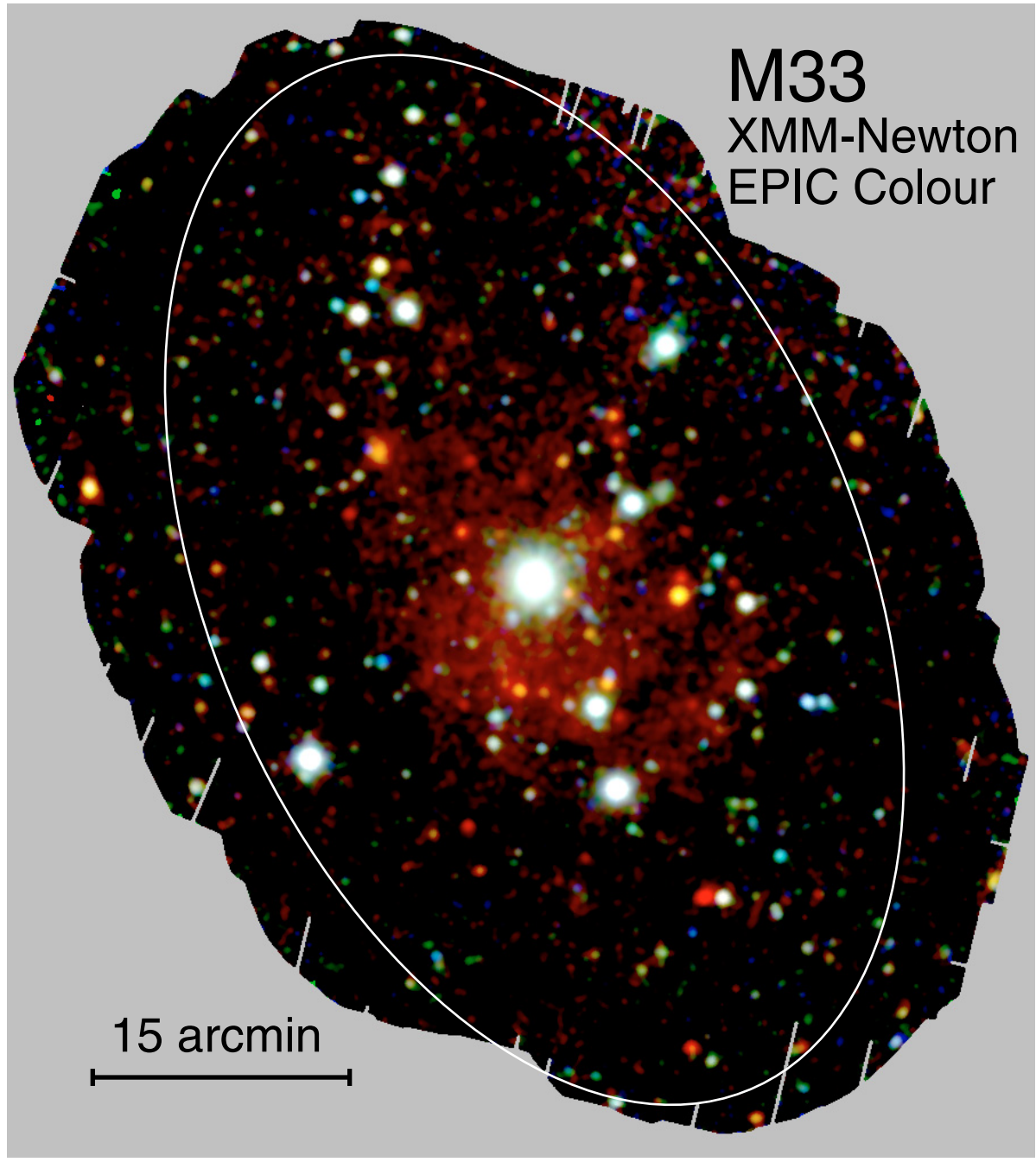

Fig. 4. Logarithmically-scaled, three-colour XMM-Newton EPIC low background image of the M 33 medium and thin filter observations combining PN and MOS1 and MOS2 cameras. Red, green and blue show respectively the $(0.2-1.0) \mathrm{keV}$, (1.0-2.0) keV and (2.0-12.0) keV bands. The data in each energy band have been smoothed with a Gaussian of FWHM 20" which corresponds to the average point spread function of the combined observations with different off-axis angles. The image has been corrected for un-vignetted exposure and masked for exposure above $14 \mathrm{ks}$. The image scale and the inclinationcorrected optical $D_{25}$ ellipse of M 33 are marked.

detected in this work) are indicated in the XID column of Table 3 (Col. 66).

From the 17 Einstein sources reported in Trinchieri et al. (1988) we detected all but the relatively bright source $X-12$ in the northern disk and the less than $3 \sigma$ detection $\mathrm{X}-15$ which was located about $90^{\prime \prime}$ to the East of the nucleus. Both of these sources were also not detected by ROSAT (Schulman \& Bregman 1995; Long et al. 1996, HP01). While one may question the original existence of $\mathrm{X}-15, \mathrm{X}-12$ was detected by Einstein with high significance and has to be strongly time variable.

In the correlation with ROSAT detected sources, we concentrated on the catalogue derived by HP01 from all archival observations of the field, which contains 184 sources within $50^{\prime}$ of the nucleus. It re-detects all 37 sources detected in the ROSAT PSPC analysis of Long et al. (1996) and 21 of the 27 sources from the first ROSAT HRI analysis of the central area of M 33 by Schulman \& Bregman (1995). We searched for these low signal-to-noise sources (numbers 18, 19, 20, 23, 24, and 27) without success in the more sensitive XMM-Newton data. The sources therefore most likely are not just time variable, but rather false detections.

In Table 4, we classified sources of HP01 who analyzed all archival ROSAT PSPC and HRI observations of the M 33 field: 19 sources are positioned outside the field covered by the XMM-Newton observations, three are detected within the extended emission of XMM-Newton source 125, 5 are resolved into multiple components in the XMM-Newton catalogue. We did not detect 41 out of 51 sources with a ROSAT detection likelihood below 10. All these sources originate from the ROSAT HRI detections list which, following the experience at the time, was generated with a likelihood limit of 8 for detection compared to the limit of 10 used for PSPC sources. If still at the ROSAT brightness, these HRI sources should have been detected by the deeper XMM-Newton observations. While, in principle, they could all have dimmed by such an amount that we did not detect them, this seems rather unlikely and probably most of these sources were spurious detections. The situation is certainly different for the HP01 sources with likelihood above 10. Many of these sources were detected with both ROSAT detectors and the non-detection with XMM-Newton indicates time variability. HP01 source 30 was already found to vary by more than a factor of 7.5 during the ROSAT observations, two other sources (69 and 82) were classified by HP01 as SSS candidates, which as a class are known to show strong time variability, and another two were classified as foreground stars (9 and 133), which might have been flaring during the ROSAT observations. The HP01 sources close to the nucleus 
Table 4. Summary of cross-correlation with the M 33 ROSAT catalogue of HP01. ROSAT sources, not detected in our XMM-Newton analysis, are arranged according to their ROSAT detection likelihood $L H$.

\begin{tabular}{ll}
\hline \hline \multicolumn{1}{c}{ Classification } & \multicolumn{1}{c}{ HP01 source numbers } \\
\hline Outside XMM-Newton field & $1,2,3,4,5,6,7,10,11,46,167,171$, \\
& $176,177,180,181,182,183,184$ \\
Within extended source 125 & $48,50,51$ \\
Resolved by XMM-Newton & $22,29,59,121,172$ \\
Not detected: $L H<9$ & $14,16,24,35,37,38,65,73,79,85,88$, \\
(28 of 34 sources) & $89,94,105,107,108,116,127,131$, \\
& $135,138,149,153,155,159,166,175$ \\
Not detected: $9 \leq L H<10$ & $23,36,43,47,56,72,90,99,100,101$, \\
(13 of 19 sources) & $113,125,142$ \\
Not detected: $L H \geq 10$ & $9(L H=29), 30(59), 41(44), 53(13)$, \\
& $68(42), 69(11), 82(27), 83(14)$, \\
& $93(198$, near nucleus), 97(11), \\
& $98(21$, near nucleus), 119(14, near \\
& nucleus), 128(18), 133(20) \\
\hline
\end{tabular}

of M 33 (93, 98, and 119) may have been missed in our XMMNewton analysis due to contamination from the bright nuclear source $\mathrm{X}-8$ or could be spurious detections due to structures in the ROSAT PSF. One other HP01 source (128) is clearly visible in the XMM-Newton band 3 and 4 images (see Fig. 3) between XMM-Newton sources 235 and 258, however it was not picked up by the detection algorithms. Besides these special cases, there are 92 clear and four questionable $(214,256,258$, 338) XMM-Newton and HP01 correlations listed in Col. 66 of Table 3.

\section{Classes of point-like X-ray sources detected in the direction of $M 33$}

To identify the M 33 X-ray sources we searched for correlations around the $\mathrm{X}$-ray source positions (within a radius of $3 \times\left(\sigma_{\text {stat }}+\sigma_{\text {syst }}\right)$ in the SIMBAD and NED archives and within several catalogues. In Cols. 67 to 72 of Table 3, we give extraction information from the USNO-B1 catalogue (name, number of objects within search area, distance, $B 2, R 2$ and $I 2$ magnitude of the brightest object). We omitted information, when the digitization was fooled by extended emission within $\mathrm{H}_{\text {II }}$ regions (mostly only some colours available and rather bright). To improve on the reliability of identifications we used the $B$ and $R$ magnitudes to calculate the ratio of X-ray-to-optical flux, given by $\log \left(\frac{f_{\mathrm{x}}}{f_{\mathrm{opt}}}\right)=\log \left(f_{\mathrm{x}}\right)+\left(m_{B 2}+m_{R 2}\right) /(2 \times 2.5)+5.37$, following Maccacaro et al. (1988, see Col. 73).

The catalogued X-ray sources are identified or classified based on properties in X-rays (hardness ratios $(H R)$, variability, extent) and of correlated objects in other wavelength regimes (Table 3, Cols. 74, 75). The criteria used are summarized in Table 5. We counted a source as identified, if at least two criteria secure the identification. Otherwise, we only counted a source as classified (indicated by pointed brackets).

We plotted X-ray colour/colour diagrams based on the HRs (see Fig. 5). Unclassified sources are only plotted if the error in both contributing $H R$ s is below 0.2 . To identify areas of specific
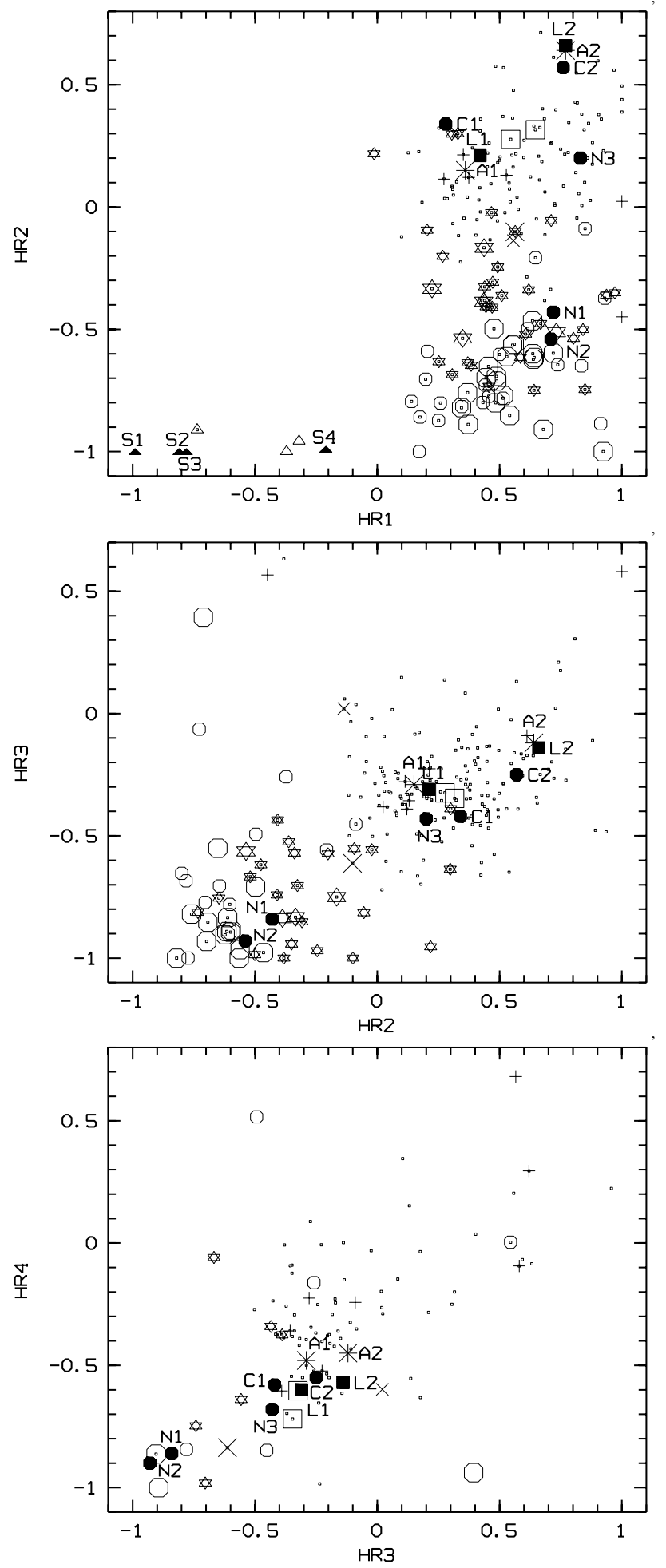

Fig. 5. Hardness ratios of sources detected by XMM-Newton EPIC. Shown as dots are only values with errors smaller than 0.20 on both $H R(i)$ and $H R(i+1)$. Foreground stars and candidate are marked as big and small stars, galaxies and candidates as big and small $\times$, AGN candidates as crosses, SSS candidates as triangles, SNR and candidates as big and small hexagons, XRBs as squares. In addition, we mark positions derived from measured XMM-Newton EPIC spectra and models for SSS (S1 to S4) as filled triangles, low mass XRBs (L1 and L2) as filled squares, SNRs (N132D as N1, 1E 0102.2-7219 as N2, N157B as N3, Crab spectra as C1 and C2) as filled hexagons, AGN (A1 and A2) as asterisk. 
Table 5. Summary of identifications and classifications.

\begin{tabular}{llrr}
\hline \hline Source type & \multicolumn{1}{c}{ Selection criteria } & Identified & Classified \\
\hline fg Star & $\log \left(\frac{f_{\mathrm{x}}}{f_{\text {opt }}}\right)<-1.0$ and $H R 2<0.3$ and $H R 3<-0.4$ or not defined & 5 & 30 \\
AGN & Radio source and not classification as SNR from $H R 2$ & 12 \\
GAL & optical id with galaxy and $H R 2<0.0$ & 1 & 1 \\
SSS & $H R 1<-0.2, H R 2-E H R 2<-0.99$ or $H R 2$ not defined, $H R 3, H R 4$ not defined & 5 \\
SNR & $H R 1>0.1$ and $H R 2<-0.4$ and not a fg Star & 21 & 23 \\
XRB & optical identification or X-ray variability & 2 & 267 \\
hard & $H R 2-E H R 2>-0.2$ or only $H R 3$ and $H R 4$ defined, and no other classification & \\
\hline
\end{tabular}

source classes in the plots, we over-plotted colours of sources, derived from measured XMM-Newton spectra and model simulations (see discussion in subsections below).

Identification and classification criteria and results are discussed in detail in the subsections on individual source classes below. Many foreground stars, SSS and SNRs can be classified or identified. However, besides a few clearly identified XRBs and AGN, and SNR candidates with known positions at other wavelengths, we have no clear hardness ratio criteria (see below) to select XRBs, Crab-like SNR or AGN. They are all "hard" sources and we therefore introduced a class $<$ hard $>$ for sources with $H R 2$ minus $H R 2$-error greater than -0.2 (see Table 5).

43 sources remained unidentified or without classification.

\subsection{Foreground stars (fg star)}

Foreground stars are a class of X-ray sources that is homogeneously distributed over the FOV of the M 33 survey. The good positioning of XMM-Newton and the available catalogue USNO-B1 allowed us to effectively select this type of sources. We identified five sources with stars of known type in the SIMBAD data base. Their X-ray fluxes were in the range expected for these sources. In addition, we classified 30 sources as foreground stars $\left(\log \left(\frac{f_{\mathrm{x}}}{f_{\text {opt }}}\right)<-1\right.$, see Maccacaro et al. (1988) and in addition $H R 2<0.3$ and $H R 3<-0.4)$. For several of the star candidates we estimated the type from the colours in the USNO-B1 catalogue using the stellar spectral flux library from Pickles (1998). For ten candidates we could not determine the type from the colours $(28,54,77,122,128,262,308$, $337,371,393)$. This may indicate that these sources are not isolated stars but more complicated systems or even, in some cases, galaxies. This expectation could, for instance, be confirmed for source 262, which was resolved into two sources in DSS2 images.

A special case was source 196, only $\sim 2^{\prime}$ WSW of the nucleus. The source correlated with 2MASS J01334186+3038491, and was catalogued by Ivanov et al. (1993) as red star 184. The X-ray HRs, optical colour and $\log \left(\frac{f_{\mathrm{x}}}{f_{\text {opt }}}\right)$ point towards a foreground star of type M6 as the identification of this source.

From the HRs, sources 7 and 10 should be foreground stars. However, there was no candidate star within the error box. As these sources were at the border of the field, the systematic error may be underestimated and nearby stars may be candidates for the optical identification.

For source 240, the HRs indicated a foreground star, however $\log \left(\frac{f_{\mathrm{x}}}{f_{\mathrm{opt}}}\right)=-0.7$, larger than the assumed limit for classification. The optical colours would indicate a M10 III star. Also the other candidate stars of type late $\mathrm{M}$ have a rather high $\log \left(\frac{f_{\mathrm{x}}}{f_{\mathrm{opt}}}\right)$.

For several sources, $\log \left(\frac{f_{\mathrm{x}}}{f_{\mathrm{opt}}}\right)$ pointed towards a stellar identification $(8,38,72,176,206,209,226,234)$. However, only for three sources the $H R$ s including errors were within the limits we assume for foreground stars $(72,176,234)$.

\subsection{Galaxies (GAL) and AGN}

Already after the Einstein observations, the X-ray bright source X-9c (297) has been identified by Christian \& Schommer (1982) with an elliptical galaxy at $z=0.03$. The XMM-Newton $\mathrm{X}$-ray luminosity of $1.35 \times 10^{41} \mathrm{erg} \mathrm{s}^{-1}(0.2-4.5 \mathrm{keV})$ was typical and the $H R$ s were compatible with an elliptical galaxy spectrum (see $H R$ plots).

From SIMBAD and NED searches, we found an additional galaxy correlation (namely source $358=$ LEDA5899).

There were no correlations with AGN with known redshift within SIMBAD or NED. We therefore only classified 12 sources as AGN based on SIMBAD, NED, and other radio source correlations (NVSS, Gordon et al. (1999)) with the additional condition of being a $<$ hard $>$ X-ray source. A final decision will only be possible using optical spectra. In Fig. 5, we included typical $H R$ s for an AGN spectrum (power law with photon index of 1.7 assuming Galactic foreground absorption $\left(6 \times 10^{20} \mathrm{~cm}^{-2}, \mathrm{~A} 1\right)$ and absorption through the densest areas of M 33 (Newton 1980, $4.8 \times 10^{21} \mathrm{~cm}^{-2}, \mathrm{~A} 2$ ).

Sources 150 and 373 could be classified as SNRs, as well as AGN. They were identified as radio sources within the inclination-corrected M $33 D_{25}$ ellipse, without optical identification as SNR by Gordon et al. (1999) and the radio spectral index did not clearly classify them as AGN.

\subsection{Super-Soft Sources (SSS)}

SSS show black body spectra with temperatures below $50 \mathrm{eV}$, they radiate close to the Eddington luminosity of a $1 M_{\odot}$ object and they are believed to be white dwarf systems steadily burning hydrogen at the surface. They were identified as a class 
of X-ray sources by ROSAT and are often observed as transient X-ray sources (see Greiner 2000, and references therein). In the catalogue, SSS were only classified using their HRs. To guide the classification, we calculated SSS HRs assuming a 25 and $50 \mathrm{eV}$ black body model spectrum assuming Galactic foreground absorption (S1 and S3) and absorption through the densest areas of M 33 (S2 and S4). This led to the selection criteria in Table 5 and the classification of five M 33 SSS $(52,109$, $223,247,332)$. They were detected with absorbed luminosities of (1-8) $\times 10^{35} \mathrm{erg} \mathrm{s}^{-1}$ in the $0.2-0.5 \mathrm{keV}$ band. Only three of the sources (and the model $H R \mathrm{~s}$ ) could be plotted in Fig. 5. For the other two, HR2 was not determined.

\subsection{Supernova remnants (SNR)}

SNRs can be identified as sources where thermal components dominate the X-ray spectrum below $2 \mathrm{keV}$ (examples are N132D in the Large Magellanic Cloud and 1E 0102.2-7219 in the Small Magellanic Cloud) and as so called "plerions" with power law spectra (examples are the Crab nebula and N157B in the Large Magellanic Cloud). To guide the classification we calculated $H R$ s from archival XMM-Newton spectra of these SNRs. Spectra of N132D (N1), 1E 0102.2-7219 (N2), and N157B (N3) could be directly compared to M 33 SNRs, as they are seen through comparable foreground absorption. For the Crab nebula spectrum, we assumed Galactic foreground absorption (C1) and absorption through the densest areas of M 33 (C2). It is clear from Fig. 5, that "thermal" SNRs are located in areas of the X-ray colour/colour plots that only overlap with foreground stars. If we assumed that we have identified all foreground star candidates from the optical correlation and inspection of the optical images, the remaining sources could be classified as SNRs with the criteria given in Table 5.

Extensive searches for SNRs in M 33 were performed in the optical (see Gordon et al. 1998, and references therein) and radio (see Gordon et al. 1999, and references therein). Many $\mathrm{X}$-ray sources in the catalogue correlated with optical and/or radio SNRs (see Table 3). We counted such sources as SNR identifications (21), if in addition they fulfilled the $H R$ criteria (see above). We counted sources as classified SNRs (23), if they either just fulfilled the $H R$ criteria (16) and were no identified or classified as foreground star, or if they correlated with optical/radio SNRs and did not fulfill the $H R$ criteria (7).

From these classified SNRs, source 20 fulfilled the $H R$ criteria, but was positioned outside the inclination-corrected optical $D_{25}$ ellipse, while sources 101 and 266 were located between two optical SNR candidates and may represent the combined emission of both. As already mentioned in the subsection on AGN, we classify sources 150 and 373 as SNR as well as AGN due to the radio correlation.

A special case was source 227 , which correlated with radio source 126 of Gordon et al. (1999), who classified it as a background source with no optical identification. The XMMNewton HRs clearly indicated a SNR nature and we therefore classified the source as a SNR.

The giant H II regions BCLMP 290 and NGC 604 were detected as extended X-ray sources (125 and 299). While the HRs of source 299 indicated a spectrum similar to thermal SNRs (several SNRs would be necessary to explain the X-ray emission), the $H R$ s of source 125 indicated a hard spectrum which may originate from several point sources (XRBs) or Crab-like SNRs. In the catalogue we only classified source 299 as a SNR.

Four additional sources in the catalogue (137, 154, 162, 280) had $H R$ s compatible with the selection criteria in Table 5, within the errors. They are good candidates for further SNR investigations in M 33 .

\section{5. $X$-ray binaries $(X R B)$}

As already mentioned in the introduction to this section, expected spectra of XRBs are similar to AGN and Crab-like SNRs. To guide the classification, we calculated $H R$ s as expected for low mass XRBs (5 keV thermal Bremsstrahlung spectrum assuming Galactic foreground absorption (L1) and absorption through the densest areas of M 33 (L2)), which show soft spectra for XRBs. As can be seen in Fig. 5 these different source classes did not separate.

Therefore we only listed the black hole XRB X-8 close to the nucleus (211) and the $3.4 \mathrm{~d}$ eclipsing HMXB X-7 (171) as identified X-ray binaries.

SIMBAD searches indicated 16 more good XRB candidates from positional coincidences with optical stars of different type in M 33 (see Table 3). Source 110 correlated with an emission line object and is a good candidate for a Be XRB, source 174 correlated with an eclipsing binary candidate (398 d period), and sources 194, 197, and 323 with variable stars. Of historical interest is the optical candidate for source 323, VHK6. This source was discussed as one of four irregular variables in the paper by Hubble (1926), in which he proposed the spiral nebula M 33 as a stellar system outside the Milky Way.

All the candidates mentioned above need further X-ray work to confirm their XRB nature.

\section{Discussion}

Besides the large number of point-like sources, there was a strong component of unresolved X-ray emission in the energy band below $2 \mathrm{keV}$. The overlay of X-ray on the DSS2 red image (Fig. 6) clearly showed the strong correlation with the inner disk and the southern and NW spiral arm. While some of this emission certainly is due to unresolved point sources, there is a significant contribution from hot gas in the disk which is more concentrated in the spiral arms and $\mathrm{H}$ in regions like NGC 604 $12^{\prime} \mathrm{NE}$ and BCLMP $29015^{\prime} \mathrm{NW}$ of the nucleus. A detailed analysis of this emission will be presented in a separate paper.

In the XMM-Newton source catalogue we presented 408 sources in the M 33 field, 35 of which could be identified or classified as foreground stars and 14 as background AGN or galaxies. As sources in M 33 we identified or classified 5 SSS, 44 SNRs, and 2 XRBs. 267 sources were classified as hard and may be either XRBs or Crab-like SNRs in M 33 or background AGN. There remained 43 sources unclassified.

We can estimate the contribution of background sources to the source catalogue extrapolating from X-ray deep field 


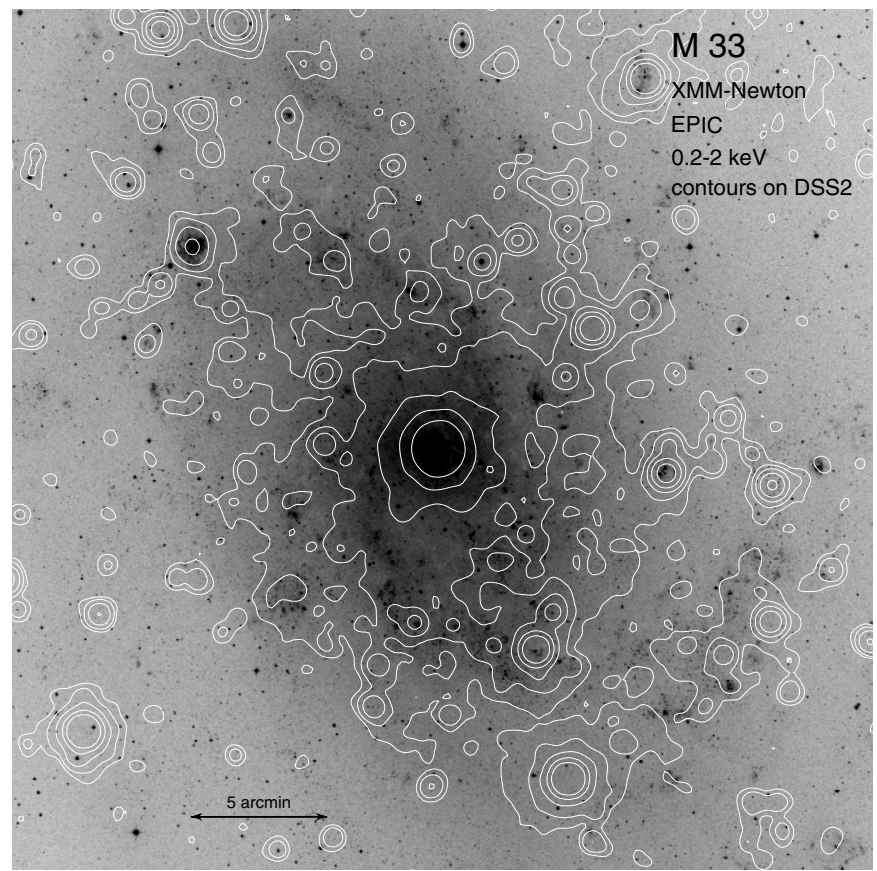

Fig. 6. XMM-Newton EPIC $0.2-2 \mathrm{keV}$ band contours of $(6,8,14,32,86) \times 10^{-5} \mathrm{ct} \mathrm{s}^{-1}$ overlayed on an optical image of M 33 extracted from the digitized sky survey (DSS2) red plates. The image scale is marked.

number counts in the $0.5-2.0 \mathrm{keV}$ and $2.0-8.0 \mathrm{keV}$ bands (see e.g. Brandt et al. 2001; Rosati et al. 2002). We used rough sensitivity maps for our survey created by the SAS task esensmap for the $0.5-2.0 \mathrm{keV}$ and $2.0-4.5 \mathrm{keV}$ bands and an estimate for additional absorption in the line-of-sight. One expects about 175 to 210 background sources detected in our field-of-view, if we assume an overlap of sources detected in the hard and soft band of $75 \%$ or only $50 \%$. Fourteen of the background sources have already been identified (see Sect. 6.2). Taking these numbers into account, up to $50 \%$ of the hard or unclassified sources should be sources within M 33 (mostly XRBs or Crab-like SNRs).

We compared the XMM-Newton source catalogue with the ROSAT catalogue of HP01. While most of the bright ROSAT sources were also detected by XMM-Newton many of the sources, only detected with the ROSAT HRI with a detection likelihood below 10, were not detected by XMM-Newton and seem to be spurious detections. From the seven ROSAT sources classified as SSS, we did not detect four. As expected for this source class, these might be transients, that were not active during the XMM-Newton observations (HP01 sources 43, 69, 76, $82)$. The remaining three seem to be false identifications: HP01 source 61 is now classified by XMM-Newton as a SNR, 84 as a foreground star and 124 as a hard source. Two ROSAT correlations with radio sources are no longer valid with the improved XMM-Newton positions (HP01 sources 63 and 147).

HP01 identified and classified 17 SNRs. The XMMNewton source catalogue marks 21 identifications and 23 classifications. While the $H R$ criteria seem to be rather robust for thermal SNRs, the identification of Crab-like remnants is doubtful and can only be secured by measurement of source extent. This may be possible with XMM-Newton EPIC for very extended SNRs (optical extent of SNR in M 33 vary from 9" to $126^{\prime \prime}$ according to Gordon et al. 1998). Most of the sources, however, will need dedicated Chandra on-axis observations for a final decision on the extent. The Chandra count rates will not be sufficient to get good spectra and a combined XMMNewton and Chandra analysis will be important for this class of sources.

The importance of combined XMM-Newton and Chandra data for M 33 XRB research was demonstrated in Pietsch et al. (2004). This type of analysis extended to all brighter sources will be the subject of a future paper.

Acknowledgements. This publication makes use of the USNOFS Image and Catalogue Archive operated by the United States Naval Observatory, Flagstaff Station (http:// www.nofs.navy.mil/data/fchpix/), of data products from the Two Micron All Sky Survey, which is a joint project of the University of Massachusetts and the Infrared Processing and Analysis Center/California Institute of Technology, funded by the National Aeronautics and Space Administration and the National Science Foundation, of the SIMBAD database, operated at CDS, Strasbourg, France, and of the NASA/IPAC Extragalactic Database (NED) which is operated by the Jet Propulsion Laboratory, California Institute of Technology, under contract with the National Aeronautics and Space Administration. The compressed files of the "Palomar Observatory Space Telescope Science Institute Digital Sky Survey" of the northern sky, based on scans of the Second Palomar Sky Survey, are copyright (c) 1993-1995 by the California Institute of Technology and are distributed herein by agreement. All Rights Reserved. Produced under Contract No. NAS 5-26555 with the National Aeronautics and Space Administration. The XMM-Newton project is supported by the Bundesministerium für Bildung und Forschung / Deutsches Zentrum für Luft- und Raumfahrt (BMBF/DLR), the Max-Planck Society and the Heidenhain-Stiftung.

\section{References}

Boulesteix, J., Courtes, G., Laval, A., Monnet, G., \& Petit, H. 1974, A\&A, 37, 33

Brandt, W. N., Alexander, D. M., Hornschemeier, A. E., et al. 2001, AJ, 122, 2810

Christian, C. A., \& Schommer, R. A. 1982, ApJ, 253, L13

Condon, J. J., Cotton, W. D., Greisen, E. W., et al. 1998, AJ, 115, 1693

Crampton, D., Gussie, G., Cowley, A. P., \& Schmidtke, P. C. 1997, AJ, 114, 2353

de Vaucouleurs, G., de Vaucouleurs, A., Corwin, H. G., et al. 1991, Third Reference Catalogue of Bright Galaxies, Vol. 1-3, XII, 2069, 7 Figs. (Berlin, Heidelberg, New York: Springer-Verlag)

Dubus, G., Charles, P. A., Long, K. S., \& Hakala, P. J. 1997, ApJ, 490, L47

Dubus, G., Charles, P. A., Long, K. S., Hakala, P. J., \& Kuulkers, E. 1999, MNRAS, 302, 731

Dubus, G., \& Rutledge, R. E. 2002, MNRAS, 336, 901

Ehle, M., Pietsch, W., \& Haberl, F. 2001, in New Century of X-ray Astronomy, ASP Conf. Ser., 251, 300

Fabrika, S. N., Sholukhova, O. N., \& Zakharova, S. A. 1997, Bull. Special Astrophys. Obs., 43, 133

Foschini, L., Rodriguez, J., Fuchs, Y., et al. 2004, A\&A, 416, 529

Freyberg, M., Briel, U., Dennerl, K., et al. 2004, in X-ray and GammaRay Instrumentation for Astronomy XIII, ed. K. A. Flanagan, \& O. H. W. Siegmund, Proc. SPIE, 5165, 112 
Giovagnoli, A., \& Mould, J. 1994, PASP, 106, 376

Gordon, S. M., Duric, N., Kirshner, R. P., Goss, W. M., \& Viallefond, F. 1999, ApJS, 120, 247

Gordon, S. M., Kirshner, R. P., Long, K. S., et al. 1998, ApJS, 117, 89

Greiner, J. 2000, New Astron., 5, 137

Haberl, F., \& Pietsch, W. 2001, A\&A, 373, 438 (HP01)

Hubble, E. P. 1926, ApJ, 63, 236

Israel, F. P., \& van der Kruit, P. C. 1974, A\&A, 32, 363

Ivanov, G. R., Freedman, W. L., \& Madore, B. F. 1993, ApJS, 89,85

Jansen, F., Lumb, D., Altieri, B., et al. 2001, A\&A, 365, L1

La Parola, V., Damiani, F., Fabbiano, G., \& Peres, G. 2003, ApJ, 583, 758

Larson, D. T., \& Schulman, E. 1997, AJ, 113, 618

Long, K. S., Charles, P. A., Blair, W. P., \& Gordon, S. M. 1996, ApJ, 466,750

Long, K. S., Dodorico, S., Charles, P. A., \& Dopita, M. A. 1981, ApJ, 246, L61

Maccacaro, T., Gioia, I. M., Wolter, A., Zamorani, G., \& Stocke, J. T. 1988, ApJ, 326, 680

Macri, L. M., Stanek, K. Z., Sasselov, D. D., Krockenberger, M., \& Kaluzny, J. 2001, AJ, 121, 870

Magrini, L., Cardwell, A., Corradi, R. L. M., Mampaso, A., \& Perinotto, M. 2001, A\&A, 367, 498

Markert, T. H., \& Rallis, A. D. 1983, ApJ, 275, 571

Massey, P. 1998, ApJ, 501, 153

ApJ, 469, 629

Massey, P., \& Conti, P. S. 1983, ApJ, 273, 576

Massey, P., Bianchi, L., Hutchings, J. B., \& Stecher, T. P. 1996,
Mochejska, B. J., Kaluzny, J., Stanek, K. Z., Sasselov, D. D., \& Szentgyorgyi, A. H. 2001, AJ, 121, 2032

Newton, K. 1980, MNRAS, 191, 615

Parmar, A. N., Sidoli, L., Oosterbroek, T., et al. 2001, A\&A, 368, 420

Peres, G., Reale, F., Collura, A., \& Fabbiano, G. 1989, ApJ, 336, 140

Pickles, A. J. 1998, PASP, 110, 863

Pietsch, W., \& Haberl, F. 2000, in The interstellar medium in M 31 and M 33, Proc., 232, WE-Heraeus Seminar, 22-25 May 2000, Bad Honnef, Germany, ed. E. M. Berkhuijsen, R. Beck, \& R. A. M. Walterbos, Shaker, Aachen, 149

Pietsch, W., Ehle, M., Haberl, F., Misanovic, Z., \& Trinchieri, G. 2003a, Astron. Nachr., 324, 85

Pietsch, W., Haberl, F., \& Vogler, A. 2003b, A\&A, 402, 457

Pietsch, W., Mochejska, B. J., Misanivic, Z., et al. 2004, A\&A, 413, 879

Regan, M. W., \& Wilson, C. D. 1993, AJ, 105, 499

Rosati, P., Tozzi, P., Giacconi, R., et al. 2002, ApJ, 566, 667

Schulman, E., \& Bregman, J. N. 1995, ApJ, 441, 568

Schulman, E., Bregman, J. N., Collura, A., Reale, F., \& Peres, G. 1993, ApJ, 418, L67

Stark, A. A., Gammie, C. F., Wilson, R. W., et al. 1992, ApJS, 79, 77

Strüder, L., Briel, U., Dennerl, K., et al. 2001, A\&A, 365, L18

Trinchieri, G., Fabbiano, G., \& Peres, G. 1988, ApJ, 329, 1037

Tully, R. B. 1988, Nearby galaxies catalog (Cambridge and New York: Cambridge University Press)

Turner, M. J. L., Abbey, A., Arnaud, M., et al. 2001, A\&A, 365, L27 van den Bergh, S. 1991, PASP, 103, 609

von Kap-Herr, A., Berkhuijsen, E. M., \& Wielebinski, R. 1978, A\&A, 62,51

Zaritsky, D., Elston, R., \& Hill, J. M. 1989, AJ, 97, 97 